\title{
OSTRACODES DE CAMARGUE
}

par F. Marazanof.

La présente note n'a pas la prétention de constituer une étude écologique approfondie des Ostracodes, mais de compléter nos connaissances sur l'inventaire des espèces de ce groupe récoltées en Camargue depuis 1962.

Je tiens à remercier le Professeur G. Hartmann pour les déterminations de ce matériel et les précieux renseignements qu'il m'a communiqués.

G. Hartmann (1953) donne une première liste d'Ostracodes des étangs méditerranéens :
Pontocypris dispar G. W. Müller
Cytherois frequens G. W. Müller
Xestoleberis auriantia Baird
Loxoconcha impressa Baird
Loxocioncha minima G. W. Müller
Loxoconcha elliptica Brady
Cyprideis littoralis Brady
Leptocythere rara G. W. Müller
Leptocythere petiti $n$. $s p$.

Parmi ces espèces, Cyprideis littoralis et Leptocythere petiti étaient fréquents en Camargue. Leptocythere petiti provenait du nord-est de l'étang du Vaccarès.

D. Schachter (1950) et Petit et Schachter (1954) signalent la répartition des espèces suivantes dans les différents milieux de Camargue :
Eucypris virens Jurine
Cypris bispinosa Lucas
Cyprideis littoralis Brady
Candona sp.
Candona candida O. F. Müller
Candona lobipes Hartw
Leptocythere petiti Hartmann
Loxoconcha elliptica Brady
Loxoconcha impressa Baird.

A cette liste il faut ajouter Pontocypris dispar G. W. Müller, espèce signalée par D. Schachter (1960) de l'étang des Impériaux et déjà mentionnée par Hartmann (1953) de l'étang du Canet. 
Depuis 1962, de nombreuses prospections dans les eaux douces et saumâtres du delta, m'ont amené à recueillir parmi les espèces invertébrées, un certain nombre d'Ostracodes non encore signalés en Camargue et nouveaux aussi pour la faune française.

Je tiens à préciser que je n'ai pas prospecté systématiquement tous les milieux de Camargue et qu'il serait intéressant d'entreprendre sur ce groupe des recherches écologiques approfondies, aussi bien dans les milieux d'eau douce qu'oligosaumâtres, saumâtres, polysaumâtres et salés (classification des eaux poikilohalines : P. Aguesse, 1957).

Ostracodes nouveaux pour la Camargue

Famille Cyprididae

subf. Cypridopsinae

Cypridopsis aculeata Costa 1847

Famille Cytheridae

subf. Paradoxostominae

Paradoxostoma cf. intermedium G. W. Müller 1894

subf. XestoleberidinaE

Xestoleberis auriantia Baird 1838

Ostracodes nouveaux pour la France

PODOCOPA

Famille Cyprididae

subf. Cypridinae

Trib. Notodromadini

Notodromas persica Gurney 1921

Trib. Erpetocypridini

Erpetocypris chevreuxi G. O. Sars

1896

Trib. Dolerocypridini

Dolerocypris sinensis G. O. Sars

1903

\section{REMARQUES ÉCOLOGIQUES}

Cypridopsis aculeata Costa

Espèce à vaste répartition biogéographique pouvant vivre dans les eaux saumâtres. Je l'ai fréquemment rencontrée en moyenne Camargue, soit dans des mares temporaires, soit dans des marais permanents. 
Station 14. Mare temporaire près des Salines de la Tour-du-Valat.

Remise en eau avec les pluies d'automne, cette mare garde un niveau élevé pendant toute la période hivernale. Au printemps d'importants herbiers de Ranunculus baudotii envahissent le plan d'eau resté libre en hiver. La population planctonique hivernale est en majorité formée par des populations du Cladocère Daphnia magna, du Diaptomide Arctodiaptomus wierzejskii et du Cyclopide Megacyclops viridis. Le 12 novembre 1963 j'ai relevé pour une salinité de 1,75 g/l et un $\mathrm{pH}$ de $7,965 \%$ de Daphnia magna, $30 \%$ d'Arctodiaptomus wierzejskii, $10 \%$ de Megacyclops viridis et $5 \%$ de Daphnia longispina. Au printemps les espèces précédentes persistent mais sont quantitativement moins bien représentées. L'Ostracode Cypridopsis aculeata forme à partir d'avril une importante population. Le 10 avril $1964(\mathrm{NaCl}=2 \mathrm{~g} / \mathrm{l}, \mathrm{pH} 8,1)$ des moyennes établies d'après mes relevés quantitatifs donnent pour cette espèce des concentrations de 120 individus/1 (Daphnia magna 55 ind./1, Arctodiaptomus wierzejskii 30 ind./1 et Megacyclops viridis 7 ind./1). En 1964 la mare s'est asséchée au mois de juin. Le 14 mai $1964(\mathrm{NaCl}=4,6 \mathrm{~g} / \mathrm{pH}=8,2) \quad$ Cypridopsis aculeata ne comptait plus que 15 ind./l. Les températures élevées et l'évaporation intense provoquent une baisse considérable des niveaux entraînant une augmentation très nette de la salinité qui passe de $6,8 \mathrm{~g} / \mathrm{l}$ le 27 mai 1964 à $14,5 \mathrm{~g} / \mathrm{l}$ le 8 juin 1964. Cypridopsis aculeata disparaît; une faune d'eau saumâtre s'installe, formée en majorité de populations benthiques : Iydrobia ventrosa, Sphaeroma hookeri, Gammarus locusta Arctodiaptomus wierzejskii, Megacyclops viridis sont remplacés par dautres espèces plus curyhalines : Calanipeda aquae dulcis ct Laophonte selosa. A une faune d'eaux oligosaumâtresmésopoikilohalines succède un peuplement d'eaux saumâtres-mésopoikilohalines (AgUESSE 1957) avec absence de Cypridopsis aculeata.

Station 2 : La Baisse Salée de la Tour du Valat.

Le cycle des invertébrés de ce marais a été bien étudié : D. Schachter (1950), P. Aguesse et L. Bigot (1960), F. MarazaNOF (1963).

Pendant le cycle biologique 1963-1964 Cypridopsis aculeata est apparu en décembre 1963 formant une population d'environ 15 ind./1 qui s'est développée pendant la biocénose de printemps où l'on comptait 45 ind./l. Une autre espèce d'Ostracode : Eucypris virens était aussi présente dans les relevés : 20 ind./l le 20 décemhre $1963(\mathrm{NaCl}=1,4 \mathrm{~g} / \mathrm{l} \mathrm{pH}=7,8), 53$ ind./l le 12 avril 1964 $(\mathrm{Nacl}=1,75 \mathrm{~g} / 1 \mathrm{pH}=8,4)$. Cypridopsis aculeata disparaît en avril. Pendant toute la durée du cycle la salinité de l'eau n'a pas dépassé $2 \mathrm{~g} / \mathrm{l}$.

Cypridopsis aculeata serait donc en Camargue une espèce d'eaux oligosaumâtres, disparaissant lorsque la salinité dépasse $5 \mathrm{~g} / \mathrm{l}$. 
Paradoxostoma cf. intermedium G. W. Müller.

Espèce marine méditerranéenne vivant parmi les algues. Trouvée dans l'étang de Beauduc le 20 octobre 1963 . $(\mathrm{NaCl}=29 \mathrm{~g} / \mathrm{l})$. Il n'est malheureusement pas possible de décrire son cycle. La présence de cette espèce dans l'étang paraît être liée aux apports d'eau de mer permanents pendant la période estivale, apports provenant de la Méditerranée et destinés à alimenter le système des salins de la Compagnie SALICAM. La salinité de ce marais se maintient aux environs de $25 \mathrm{~g} / 1$ en hiver pour atteindre au printemps et en été 35 à $40 \mathrm{~g} / 1$.

\section{Xestoleberis aurianta Baird.}

Espèce signalée par G. Hartmann (1953) de l'étang de Salses, connue également des mers du nord. Non encore signalée de Camargue où elle vit dans l'étang du Fournelet. Espèce typique des eaux saumâtres vivant parmi les algues. Trouvée en abondance dans cet étang de mai à septembre, elle est capable de supporter d'importantes variations de salinité et des températures élevées (voisines de 30 à $35^{\circ}$ en août 1964). En avril 1964 la salinité atteignait 6 à $7 \mathrm{~g} / \mathrm{l}$ environ, passant progressivement à $10 \mathrm{~g} / 1$ en juin et $20 \mathrm{~g} / 1$ en août 1964 (valeurs sensiblement analogues en 1963). Quantitativement l'espèce est bien représentée et sa distribution est liée à la présence d'algues comme Chaetomorpha linum, Monostroma oxycoccum, Enteromorpha intestinalis, Polysiphonia flocculosa qui forment des accumulations éparses. Le maximum d'individus s'observe en juillet (moyennes de 350 ind./1 le 20 juillet 1964 dans des Chaetomorpha, $\mathrm{NaCl}=15,5 \mathrm{~g} / 1 \mathrm{pH}=7,9)$.

\section{Notodromas persica Guerney.}

Dolerocypris sinensis G. O. Sars.

Ces deux espèces proviennent d'un prélèvement dans une roubine du nord de la Camargue, à proximité du pont de St-Gilles. Cette roubine est alimentée par des écoulages de cultures irriguées : rizières en particulier. La salinité ne dépassait pas $0,5 \mathrm{~g} / \mathrm{l}$ le 29 juin 1963. Notodromas persica avec $55 \%$ des individus était l'espèce la mieux représentée, Dolerocypris sinensis ne formant que $20 \%$ des individus, Candona sp. $12 \%$, Cyclocypris sp. $13 \%$. Ces espèces vivaient avec des formes banales dulçaquicoles de Copépodes : Eucyclops serrulatus, Acanthocyclops robustus, Megacyclops viridis et de Cladocères : Simocephalus vetulus, Daphnia pulex, Ceriodaphnia reticulata.

J'ai retrouvé Notodromas persica pendant la biocénose d'automne (24-11-1963, $\mathrm{NaCl}=0,5, \mathrm{pH}=7,9)$ dans le marais de l'Esquineau de la Tour du Valat (marais oligosaumâtre-oligopoïkilohalin) avec une population abondante d'Eucypris virens et de Cypris bispinosa. 


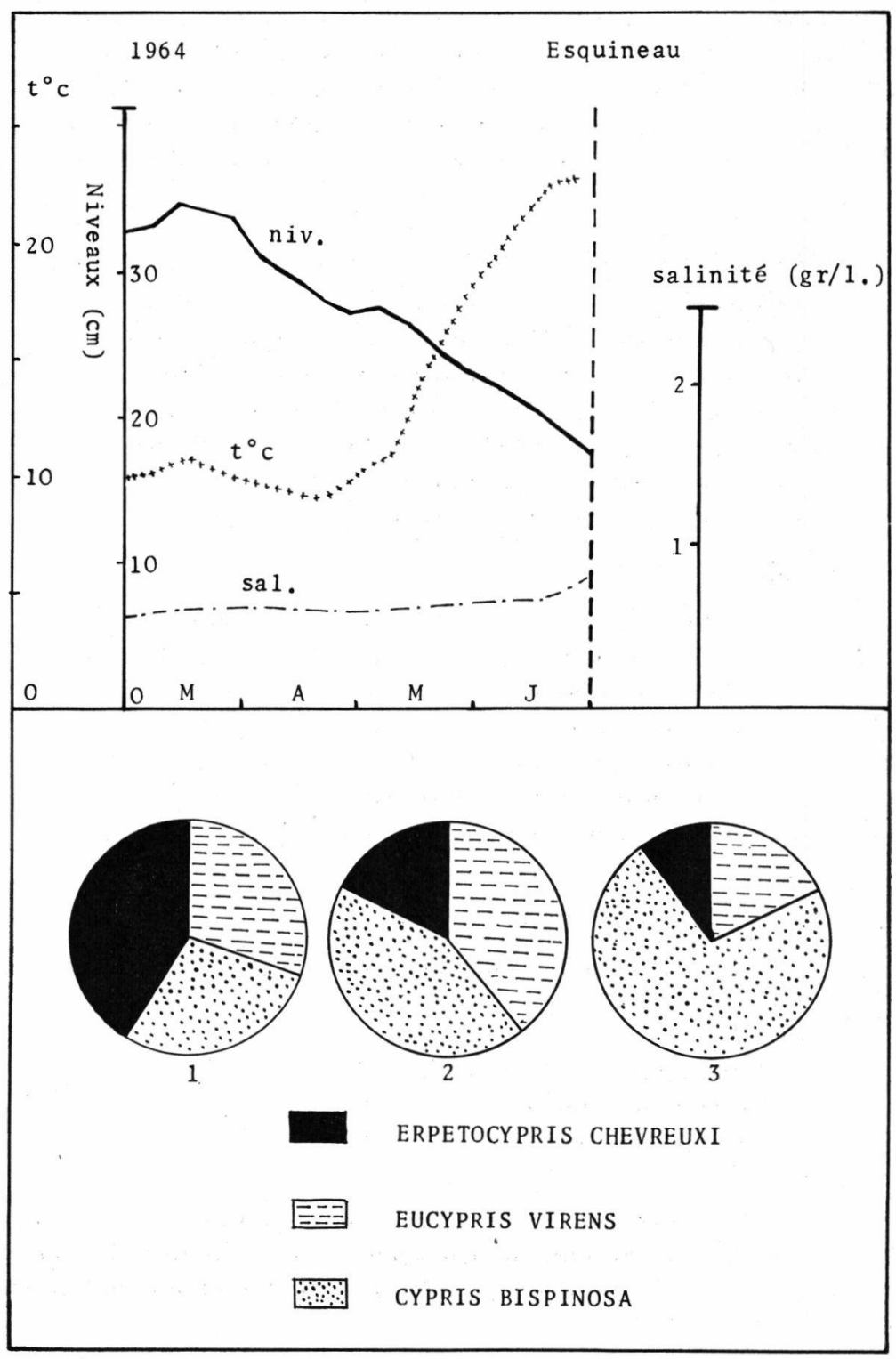

FIG. 1. - Marais de l'Esquineau. Evolution des niveaux, températures et salinité (gr. de $\mathrm{NaCl} / \mathrm{l}$.) de l'Esquineau, de mars à juin 1964. Au-dessous. Composition des populations d'Ostracodes (\% d'individus) en mars, avril et mai 1964. 
Dolerocypris sinensis et Notodromas persica sont cités pour la première fois en France. Selon une communication personnelle du Professeur G. HartmanN, ces deux espèces étaient connues de l'Italie du Nord ou plus précisément des Balkans. Il est intéressant de noter au point de vue biogéographique leur avancée vers l'ouest de l'Europe. Espèces dulçaquicoles capables de supporter de faibles salinités.

Erpetocypris chevreuxi G. O. Sars.

Espèce nouvelle pour la faune française, connue de divers milieux méditerranéens. Vit dans les eaux douces ou oligohalines. Trouvée en Camargute dans le marais de l'Esquineau où la salinité ne dépasse jannars $3 \mathrm{~g} / \mathrm{l}$ de $\mathrm{NaCl}$ (fig. 1). Fait partie de la biocénose de printemps (Marazanor 1964) avec Cypris bispinosa et Eucypris virens. Le 10 mars $1964(\mathrm{NaCl}=0,5 \mathrm{~g} / \mathrm{l} \mathrm{pH}=8)$ la proportion des espèces de la biocénose était la suivante :

Clabocènes

$\begin{array}{lrc}\text { Daphnia pulex } & 6 \% \text { des } & \text { individus } \\ \text { Acroperus harpae } & 6 \% & -- \\ \text { Chydorus sphaericus } & 15 \% & -- \\ \text { Pleuroxus laevis } & 4 \% & -- \\ \text { Pleuroxus aduncus } & 8 \% & - \\ \text { Ceriodaphnia laticaudata } & 10 \% & - \\ \text { Simocephalus vetulus } & 15 \% & -\end{array}$

CopéPOdES

$\begin{array}{lrl}\text { Megacyclops viridis } & 10 \% & - \\ \text { Diacyclops bicuspidatus } & 6 \% & -\end{array}$

OSTRACODES

$\begin{array}{lrl}\text { Erpetocypris chevreuxi } & 9 \% & - \\ \text { Cypris bispinosa } & 12 \% & - \\ \text { Eucypris virens } & 9 \% & -\end{array}$

Le 20 avril 1964, j’ai relevé des concentrations moyennes de 20 ind./l de Cypris bispinosa, Eucypris virens 15 ind./l, Erpetocypris chevreuxi 6 ind./l. Fin mai, début juin, Erpetocypris chevrenxi disparaît.

Parmi les 6 espèces d'Ostracodes non encore signalées de Camargue 3 sont nouvelles pour la France. Dolerocypris sinensis et Notodromas persica n'étaient connues que des Balkans et de l'Italie du Nord. Elles sont localisées principalement dans des eaux homoiohalines ou faiblement oligohalines de la haute et moyenne Camargue. Erpetocypris chevreuxi serait d'après G. HartmanN une espèce méditerranéenne, introduite plus au nord par les oiseaux. Cette 
espèce est aussi dulçaquicole, capable de persister dans des milieux oligosaumâtres-oligopoikilohalins.

Cypridopsis aculeata disparaît lorsque le cycle de la salinité de certains marais dépasse $5 \mathrm{~g} / \mathrm{l}$ de $\mathrm{NaCl}$.

Xestoleberis auriantia : espèce euryhaline trouvée en moyenne Camargue est typique des eaux saumâtres.

Paradoxostoma cf. intermedium signalée de Beauduc dans la zone salée des étangs inférieurs est une espèce marine, capable de vivre dans des lagunes où la salinité est voisine de celle de la Méditerranée.

\section{RÉSUMÉ}

L'auteur mentionne 6 espèces nouvelles pour la Camargue : Cypridopsis aculeata, Paradoxostoma cf. intenmedium, Xestoleberis auriantia, Notodromas persica, Erpetocypris chevreuxi, Dolerocypris sinensis. Les 3 dernières espèces sont nouvelles pour la faune française.

Dolerocypris sinensis, Notodromas persica sont localisés dans la haute Camargue dans des biotopes d'eau douce, ou faiblement oligohalins.

Erpetocypris chevreuxi serait aussi une espèce d'eau douce capable de résister à de faibles salinités.

Cypridopsis aculeata se rencontre dans des milieux oligosaumâtresoligopoikilohalins.

Xestoleberis auriantia pullule dans les eaux saumâtres.

Paradoxostoma cf. intermedium : espèce marine, est capable de vivre dans les lagunes du littoral où la salinité varie de 30 à $40 \mathrm{~g} / \mathrm{l}$ de $\mathrm{NaCl}$.

Il est intéressant de noter l'avancée vers l'ouest de l'Europe de Dolerocypris sinensis et de Notodromas persica, espèces connues des Balkans et de l'Italie du Nord.

The author has found 6 species new to the Camargue : Cypridopsis aculeata, Paradoxostoma cf. intermedium, Xestoleberis auriantia, Notodromas persica, Erpetocypris chevreuxi, Dolerocypris sinensis. The three last species are new to the French fauna. Dolerocypris sinensis, Notodromas persica are localized in the upper and middle Camargue in fresh and slightly oligohaline waters. Erpetocypris chevreuxi is also a freshwater species able to resist low salinity. Cypridopsis aculeata is found in oligopoikilohaline waters. Xestoleberis auriantia is very abundant in brackish waters. Paradoxostoma cf. intermedium : a marine species, is able to live in littoral lagoons where the salinity varies from 30 to $40 \mathrm{~g} / \mathrm{l}$. NaCl.

It is interesting to note the extension of the range of Dolerocypris sinensis and Notodromas persica, previously known only in the Balkans and Northern Italy.

Der Verfasser erwähnt sechs Arten, die neu für die Camargue sind : Cypridopsis aculeata, Paradoxostoma cf. intermedium, Xestoleberis auriantia, Notodromas persica, Erpetocypris chevreuxi, Dolerocypris sinensis. Die letzten drei sind neu für die Fauna von Frankreich. Dolero- 
cypris sinensis, Notodromas persica kommen nur in der hohen und mittleren Camargue vor, und zwar in Biodonosen süsser oder leicht salzhaltiger Gewässer. Es ist wahrscheinlich, dass Erpetocypris chevreuxi auch eine Süsswasserart ist, die einem leichten Salzgehalt widerstehen kann.

Cypridopsis aculeata findet sich in oligopoikilohalinen Gewässern.

Xestoleberis auriantia ist sehr haüfig in Brackwässern.

Paradoxostoma cf. intermedium : eine Art, die im Meer vorkommt, besitzt die Fähigkeit in Küstenlagunen zu leben, wo der Salzgehalt zwischen 30 bis $40 \mathrm{~g} / \mathrm{l} \mathrm{NaCl}$ schwankt.

Es ist interessant festzustellen, dass man im westlichen Europa die beiden Arten Dolerocypris sinensis und Notodromas persica gefunden hat, die bisher auf dem Balkan und in Norditalien bekannt waren.

\section{BIBLIOGRAPHIE}

Aguesse (P.). 1957. La classification des eaux poikilohalines, sa diffculté en Camargue, nouvelle tentative de classification. Vie et Milieu, 8 : 341-363.

Aguesse (P.) et Bigot (L.). 1960. Observations floristiques et faunistiques sur un étang de moyenne Camargue : la Baisse-salée de la Tour du Valat. Vie el Milieu, $8: 284-307$.

Hartmann (G.). 1953. Ostracodes des étangs méditerranéens. Vie et Milieu, $4: 707-712$.

Marazanof (F.). 1963. Cycle annuel des populations de Cladocères et Copépodes du Saint-Seren, de la Baisse Salée, des Relongues et Cerisières de la Tour du Valat. La Terre et la Vie, 3 : 335-356.

Marazanof (F.). 1964. Cladocères nouveaux pour la Camargue. La Terre et la Vie, 3 : 380-382.

Petit (G.) et Schachter (D.). 1954. La Camargue. Etude écologique et faunistique. Année Biol., 20 : 193-253.

Schachter (D.). 1950. Contribution à l'étude écologique de la Camargue. Ann. Inst. Océan., 25 : 1-108.

Schachter (D.). 1960. Données nouvelles sur l'évolution de quelques étangs du delta du Rhône. Comm. int. expl. sci. Mer Méảit. Monaco, 15, 3 : 155-162.

(Station Biologique de la Tour-du-Valat

et Laboratoire de Zoologie,

Faculté des Sciences de Toulouse.) 